Supporting Information

\title{
Biomimetic Nonuniform, Dual-Stimuli Self-Morphing Enabled by Gradient Four-Dimensional Printing
}

Zhengyi Song ${ }^{l}$, Luquan Ren ${ }^{l}$, Che Zhao ${ }^{2}$, Huili Liu ${ }^{l}$, Zhenglei Yu ${ }^{l}$, Qingping Liu ${ }^{l, *}$, Lei Ren ${ }^{l, 3 *}$

1. Key Laboratory of Bionic Engineering (Ministry of Education), Jilin University, Changchun 130022, China

2. Institute of Biomedical Engineering and Health Sciences, Changzhou University, Changzhou 213164, China

3. School of Mechanical, Aerospace and Civil Engineering, University of Manchester, Manchester, M13 9PL, UK

*Corresponding Author: *E-mail: liuqp@jlu.edu.cn (Q.L.)

*E-mail: lei.ren@manchester.ac.uk (L.R.) 


\section{Supporting Text}

Text 1

Material properties of PUs. The attenuated total reflectance Fourier transform infrared spectroscopy (FTIR-ATR, Nicolet Nexus FTIR spectrophotomer, USA) were conducted to compare the differences between two PU elastomers. Fig. S1 shows the FTIR spectrum of two PU elastomers. The water-swellable PU has more broader and stronger stretching bands at 1016 $\mathrm{cm}-1$ because of the presence of a large amount of hydrophilic $\mathrm{C}-\mathrm{O}-\mathrm{C}$ ether groups, endowing the PU material with water swelling capability.

The mechanical properties of two PUs were measured using a precision test system (ZQ-990A, China). The dimensions of the specimens are about $30 \mathrm{~mm} \times 10 \mathrm{~mm} \times 2 \mathrm{~mm}$. The obtained stress-strain curves of test samples are shown in Fig. S1a. Elastic moduli are determined by the fitting of initial linear region, shown in Fig. S1b, from which we can conclude that the moduli of two PUs are relatively close.

Text 2

Controllability of swelling ratio. The distribution of the swelling ratio determines the deformation of the printed structure. It can be known from Figure S2 that the linear expansion ratio of the water-swellable PU is about $150 \%$, which helps to predict the length change after deformation. As shown in Figure S4, we design three square sheets with different gradient patterns and side length $L$. According to the distribution of the swelling ratio, the deformation results can be predicted. For the square sheet shown in Figure S4a, the water swelling ratio is linearly distributed from 0 to the highest. The shape after deformation should be an isosceles 
trapezoid. Theoretically, the length of the side with the largest water swelling ratio should become $1.5 \mathrm{~L}$, and the length of its two waists with linear pattern from 0 to the highest should become 1.25L. For the square sheet with saddle function distribution shown in Figure S4b, due to the central symmetry property, it is likely to infer that the shape after deformation should be a diamond. Because each side is a linear distribution from 0 to the highest, the side length would become $1.25 \mathrm{~L}$. As for the linearly distributed water swelling ratio along the diagonal direction shown in Figure S4b, according to the linear distribution pattern on each side, the length of the two adjacent short-sides of the quadrangle after deformation would become $1.125 \mathrm{~L}$, and the other two long-sides would become 1.375L.

Without considering the out-of-plane buckling deformation, the deformed results of the three design patterns are well consisted with the as-predicted results. The overall shape of the quadrangle after deformation is in common with the predicted, and the length of the change in every side length is also basically in same as the predicted length. The investigation results proves that the gradient 4D printing method proposed in this paper has the capability to distribute the water swelling ratio by regulating the fraction of the two elastomers.

Text 3

Mathematical model. Based on Timoshenko's theory, the final curvature $K$ of a deformed hygromophic bilayer can be expressed as

$$
K=\frac{1}{\rho}=\frac{6(1+m)^{2}}{3(1+m)^{2}+(1+m n)\left(m^{2}+1 / m n\right)} \frac{\Delta \alpha \Delta \phi}{h}
$$


where $\rho$ represents the radius of curvature, $\Delta \alpha$ is the differential swelling ratio of the two layers, $m$ and $h$ is the ratio and sum of the layer thicknesses, $n$ is the ratio of the moduli of two layers, and $\Delta \phi$ is the difference of relative humidity.

This quasi-static theory is also applicable to the printed PU bi-layers with varying swelling ratio. In experiments, only $\alpha$ is in change while other parameters remained unchanged. Assuming that the dynamic change in the volume fraction of swellable PU only changes swelling ratio, and has no any effect on material modulus, the above equation can be simplified as

$$
K=\frac{1}{\rho}=k \cdot \Delta \alpha
$$

where $k$ is a constant written as

$$
k=\frac{6(1+m)^{2}}{3(1+m)^{2}+(1+m n)\left(m^{2}+1 / m n\right)} \frac{\Delta \phi}{h}
$$

Consequently, the final curvature of a certain point is only linearly proportional to $\Delta \alpha$ of this point.

According to the formula for calculating the curvature $K$ of the arbitrary point on a given curve $y=f(x)$

$$
K=\frac{1}{\rho}=\frac{y^{\prime \prime}}{\left(1+y^{\prime 2}\right)^{3 / 2}}
$$

where $y^{\prime}$ and $y^{\prime \prime}$ are the first and second derivatives of y respectively.

Considering equation (2), the equation defining the final deformed shape of the PU bi-layer can be expressed as

$$
k \cdot \Delta \alpha=\frac{y^{\prime \prime}}{\left(1+y^{\prime 2}\right)^{3 / 2}}
$$


When $K$ is constant in equation (12), the primitive function $y$, that is, the shape of the deformed object, can be derived as

$$
y=-\frac{1}{K} \sqrt{1-(K x+a)^{2}}+b
$$

where $a$ and $b$ are two constants to be determined.

For a general case when $K$ varies along the length of the object, we can consider the deformed curve is approximately formed by a number of piecewise arcs with a very small interval $\Delta x$. The shape of $i_{t h}$ piecewise arc can be defined as

$$
y_{i}=-\frac{1}{K_{i}} \sqrt{1-\left(K_{i} x_{i}+a_{i}\right)^{2}}+b_{i}
$$

where $K_{i}$ is the curvature of the $i_{t h}$ piecewise arc, $a_{i}$ and $b_{i}$ are two constants to be determined.

Text 4

Configuration Fitting. Harnessing equation (7) can enable us approximate the shapes of the deformed bi-layer objects. First, the piecewise radius of curvature $\rho_{i}$ can be determined based on local material information $\Delta \alpha_{i}$

$$
\rho_{i}=\frac{1}{K_{i}}=\frac{1}{k_{i} \cdot \Delta \alpha_{i}}
$$

Thereafter, the radian value $\theta_{i}$ of a piecewise arc with an infinitely small interval $\Delta x$ can be calculated as 


$$
\theta_{i}=\frac{l_{i}}{\rho_{i}} \approx \frac{\Delta x}{\rho_{i}}
$$

where $l_{i}$ is the arc length of the $i_{\text {th }}$ piecewise segment ${ }^{[1]}$ (Figure S15). Here, we define the length of $\Delta x$ as $0.1 \mathrm{~mm}$.

Using this method, we can approximate the shape-morphing curves from $1 \mathrm{D}$ to $2 \mathrm{D}$ for the printed bi-layer structures based on the pre-defined swelling ratio distributions. Assuming that the leftmost endpoint remains stationary with the coordinate of $(0,0)$, and the arc of first segment opens upward and is tangent to the $\mathrm{X}$ axis, the starting and ending coordinates of each arc can be calculated as

$$
\begin{aligned}
& x_{i}=x_{i-1}+\Delta x_{i}=x_{i-1}+\rho_{i} \sin \sum \theta_{i}-\rho_{i} \sin \sum \theta_{i-1} \\
& y_{i}=y_{i-1}+\Delta y_{i}=y_{i-1}+\rho_{i} \cos \sum \theta_{i-1}-\rho_{i} \cos \sum \theta_{i}
\end{aligned}
$$

The deformed shapes can be obtained by smoothly connecting each point of $\left(x_{i}, y_{i}\right)$. Notably, this method does not take into account the elongation in length.

In detail, we need define the minimum radius of curvature first, then, the changing patterns of $\rho$ and $\theta$ can be acquired based on equation (8) and (9). According to the calculation method of formula (10) and (11), the coordinates of each point on the final shape can be sequentially obtained. Finally, The terminal shape can be obtained by smoothly connecting these points. The shapes obtained by the calculation models shown in Figures 3 and 4 are all acquired in this way. 
Supporting References:

[1] M. Rüggeberg, I. Burgert, Plos One 2015, 10, 0120718 . 


\section{Supporting Figure}

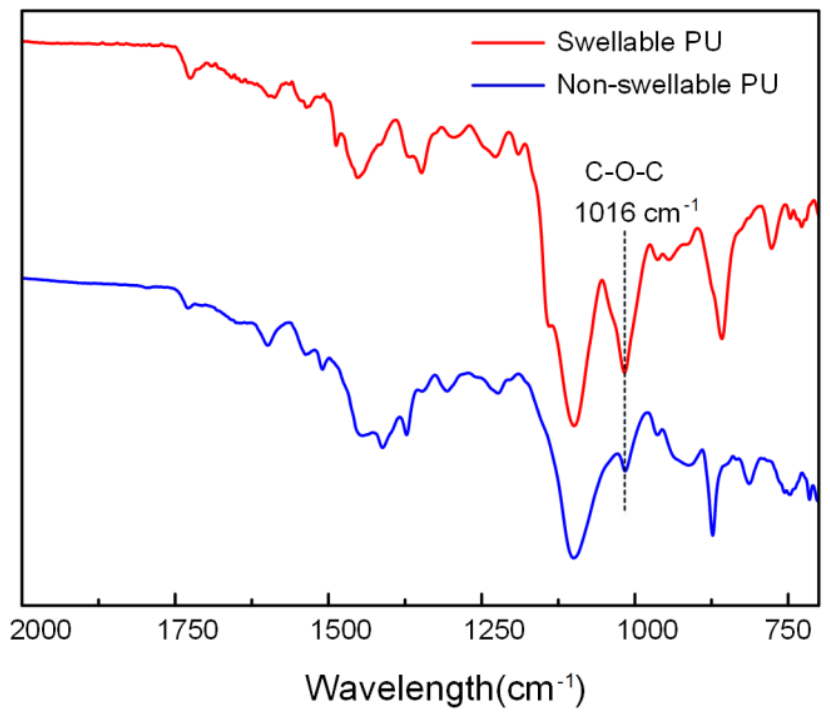

Figure S1. FTIR-ATR spectra of two PU elastomers. Red represents water-swellable PU, and blue represents non-swellable one.

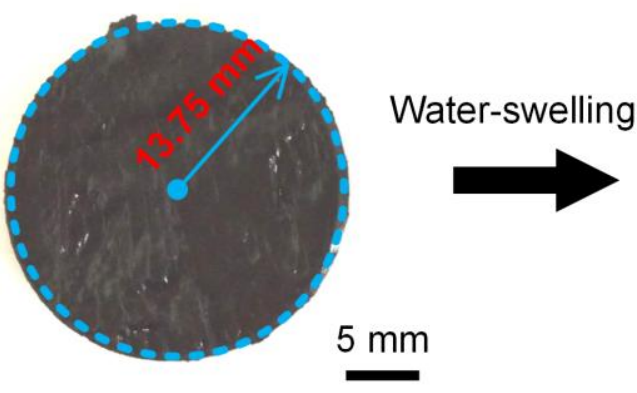

Before shape-shifting

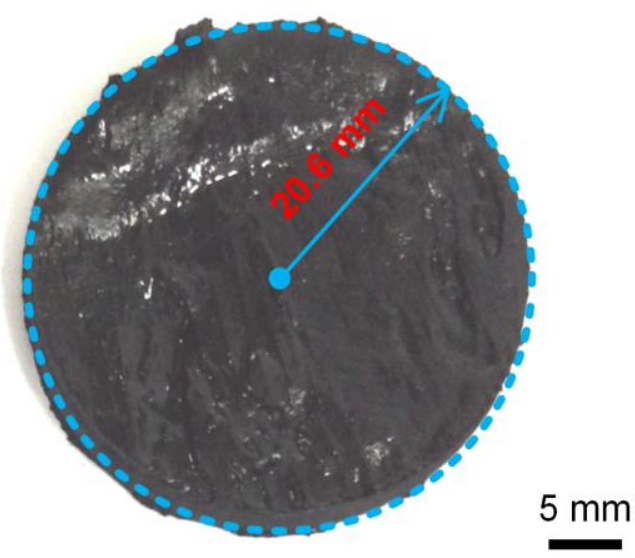

After shape-shifting

Figure S2. The volume change of the water-swellable PU materials after water swelling. 

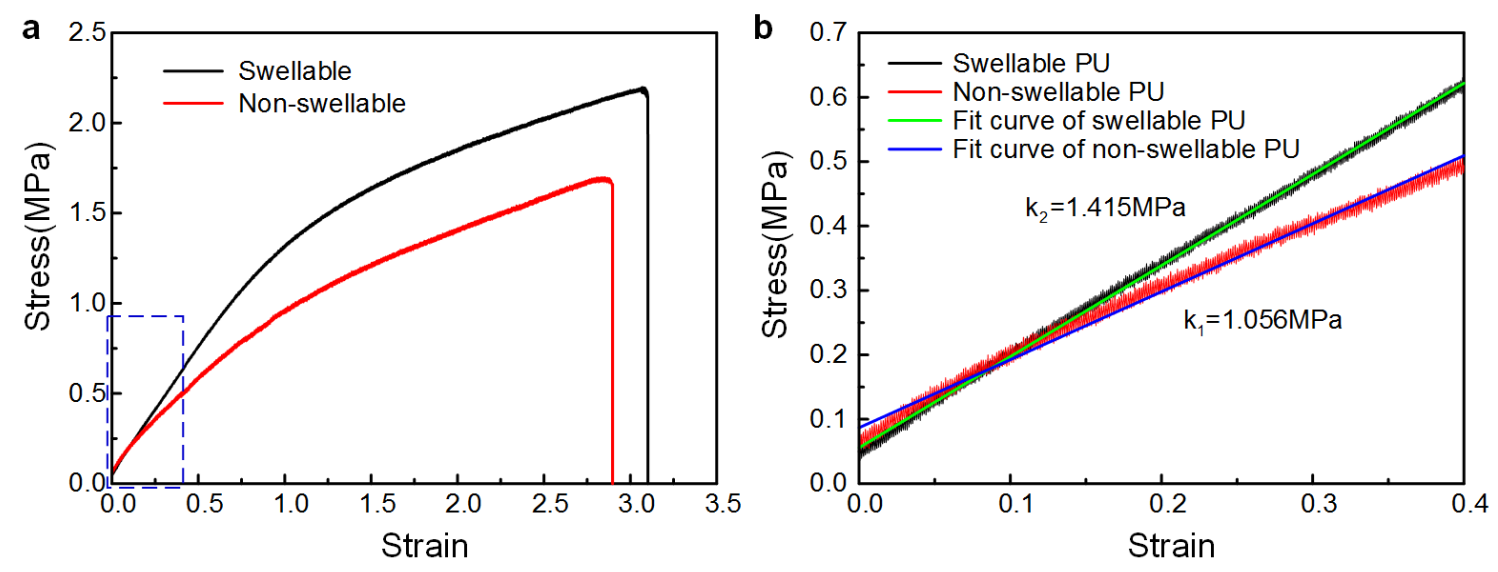

Figure S3. (a) Tensile stress-strain curves of two PU elastomers. (b) Initial linear regions of stress-strain curves and their linear fitting curves.

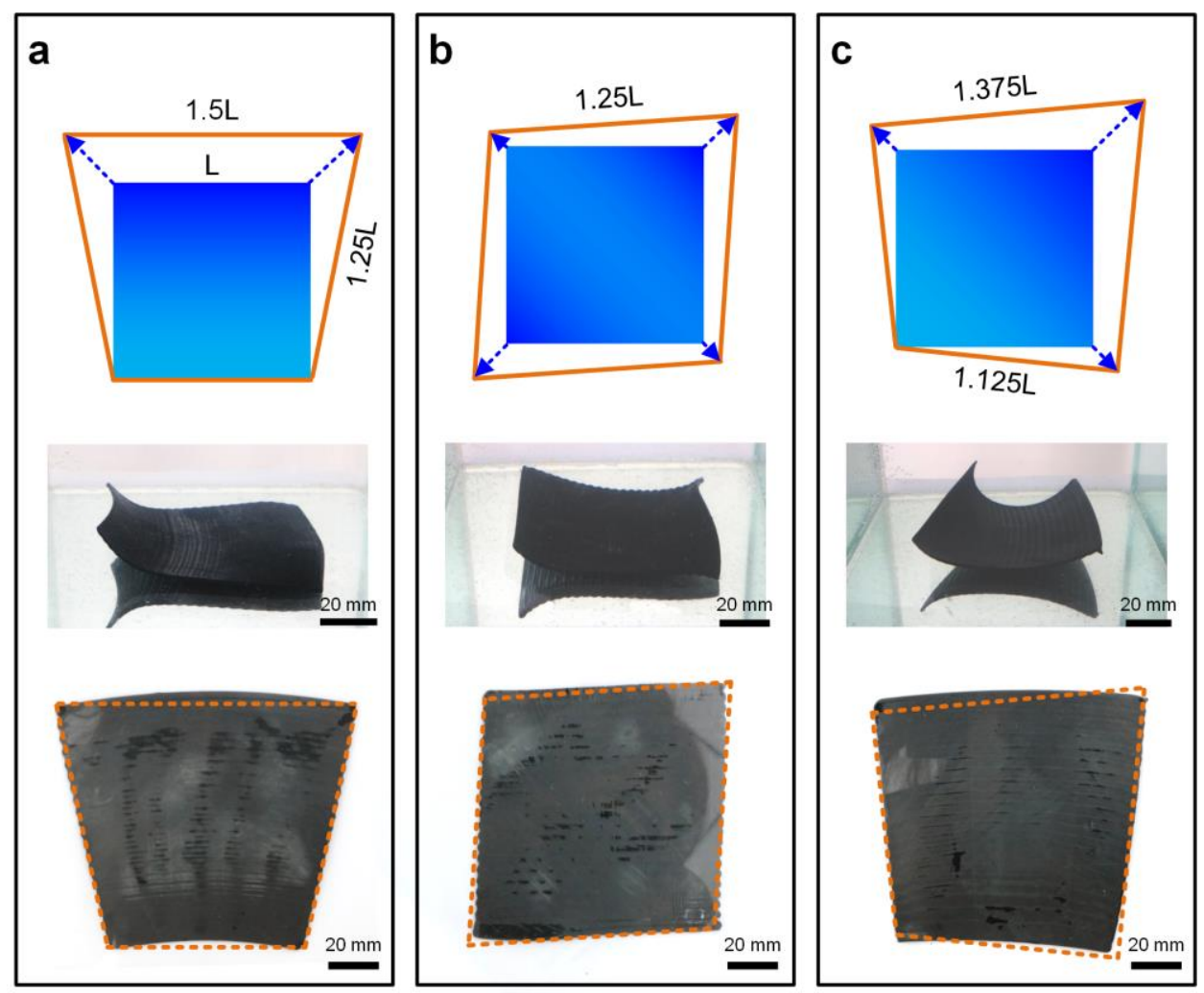

Figure S4. Printing of single layer square sheets with varying swelling ratio. The tops are swelling ratio distributions and theoretically predicted shape (orange lines), the middles are the 
actual deformed shapes after water immersion, and the bottoms are the manually flattened sheets of swollen structures compared with geometric predictions (a) Linearly graded swelling ratio along vertical direction, that morphs into an isosceles trapezoid. (b) Saddle-like swelling ratio distribution, that transforms into a rhombus. (c) Linearly distributed swelling ratio along the diagonal direction, that evolves into a trapezium.
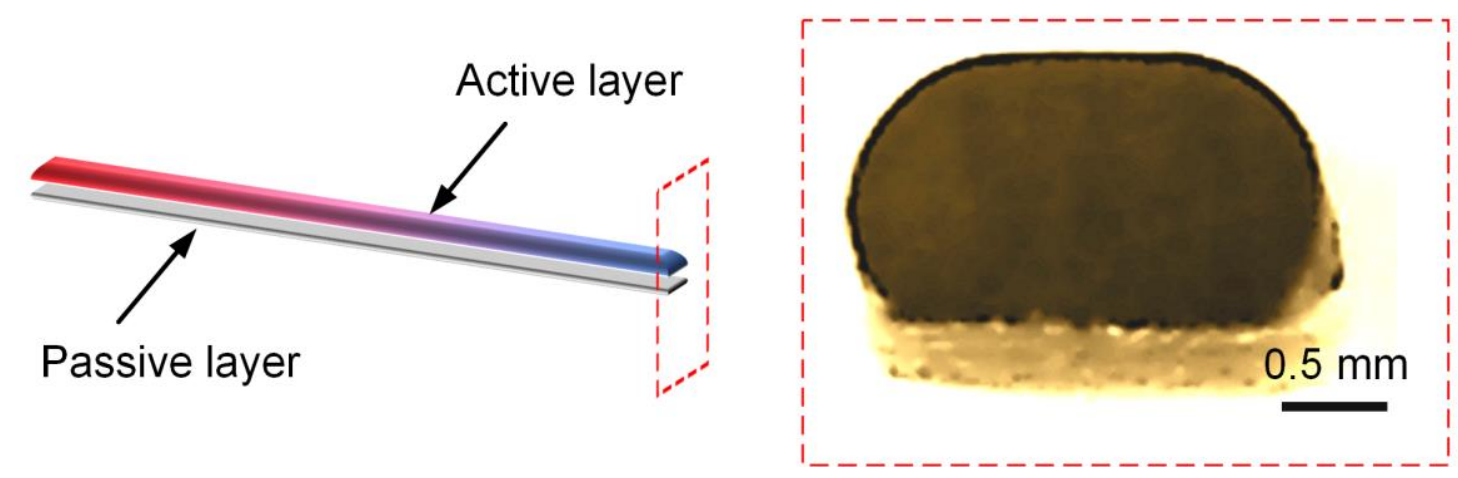

Figure S5. Cross section of the printed single PU filament onto paper.

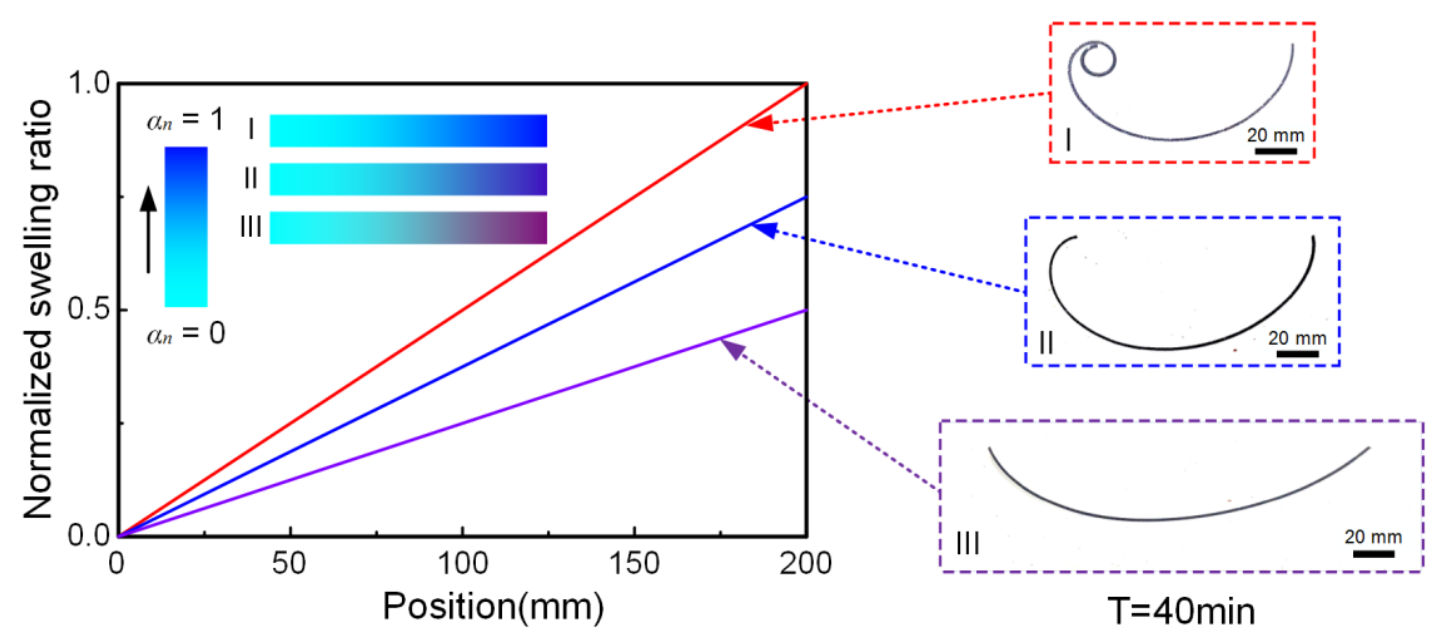

Figure S6. Effect of change rate of swelling ratio along the length on shape-morphing amplitude in PU/paper bilayer structures. (I), (II) and (III) correspond to maximum varying rate (from $0 \%$ to $100 \%), 75 \%$ of maximum varying rate and $50 \%$ of maximum varying rate respectively. 


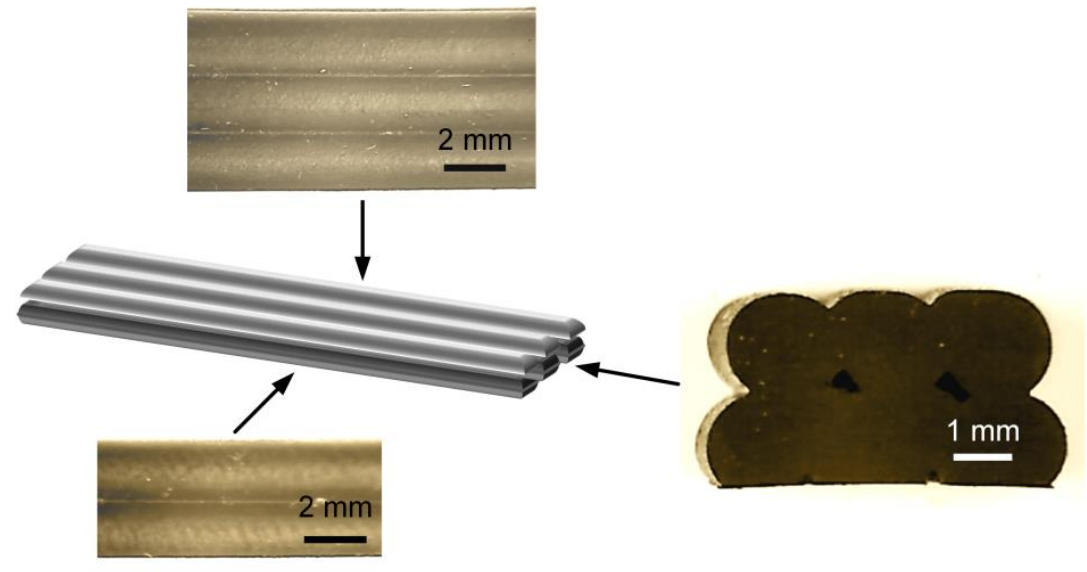

Figure S7. Top view, side view and cross section of a printed bi-layer PU strip.
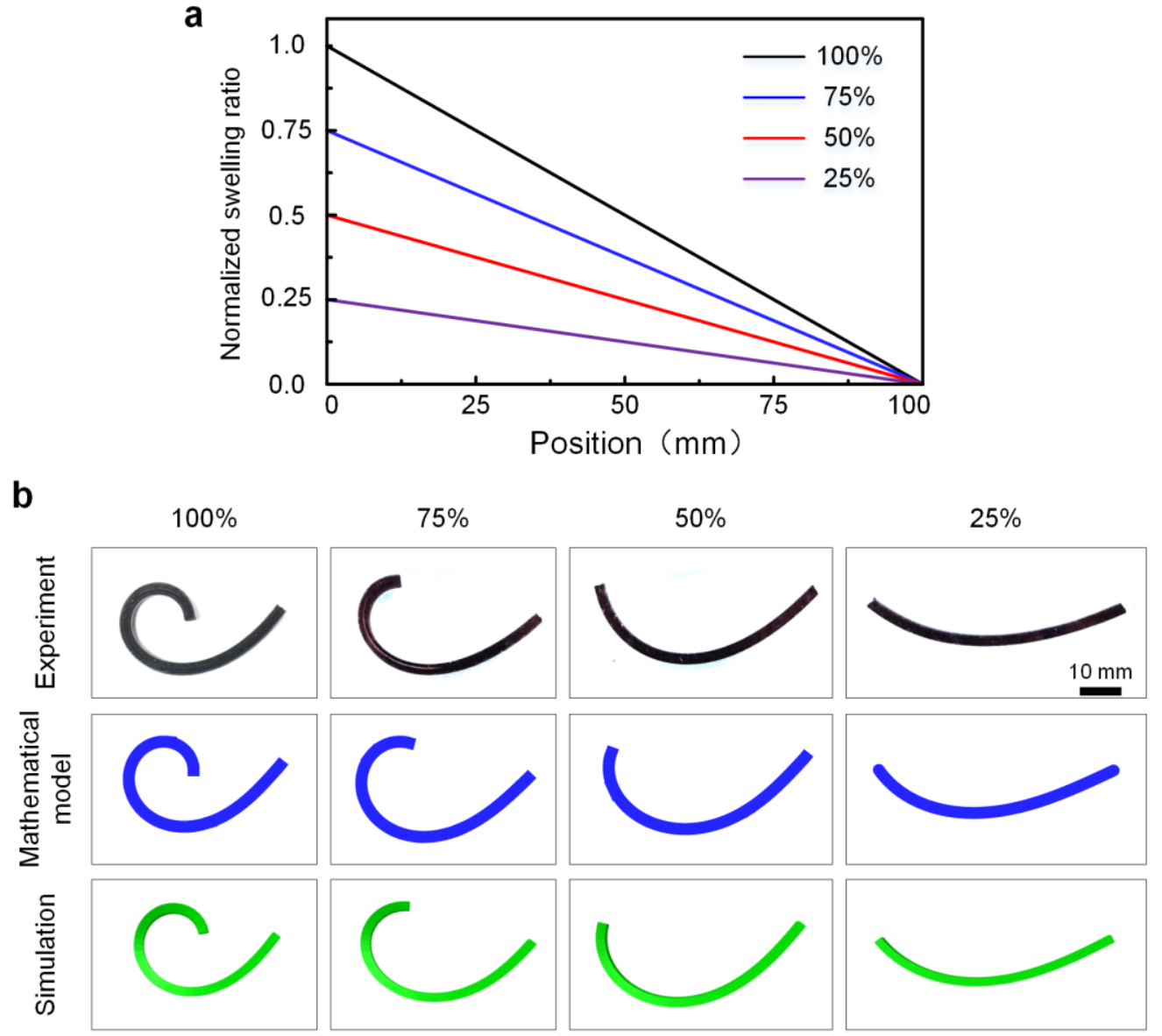
Figure S8. (a) Different linear variation designs in active layer. (b) Spiral shapes obtained by experiment, mathematical model, and simulation under different linear variation designs, respectively.

a
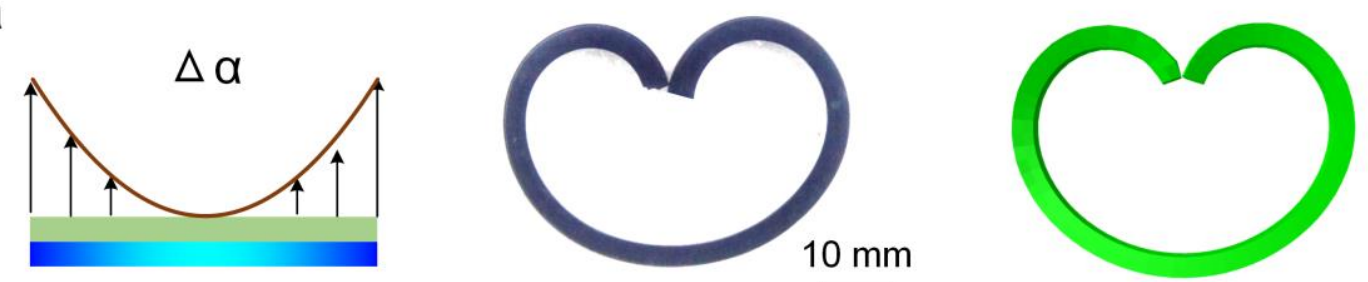

b
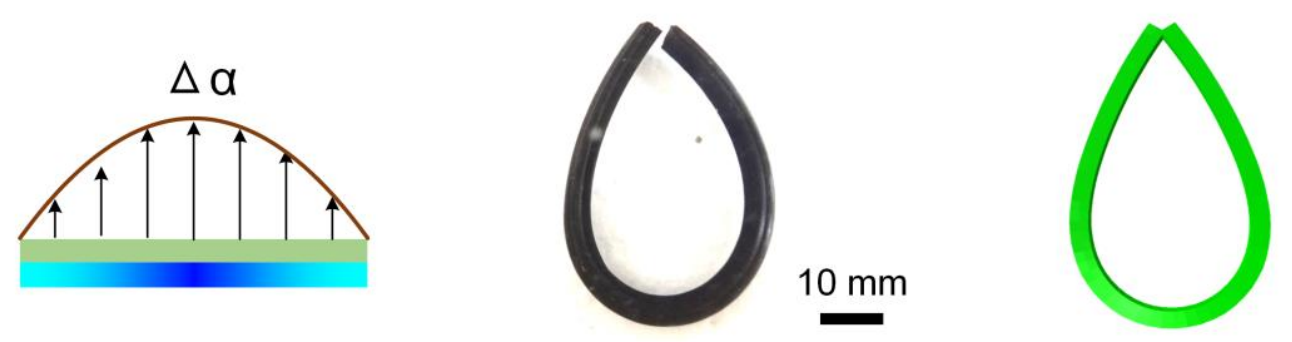

Figure S9. (a) Heart-shaped pattern generated by swelling a structure that swelling ratio is distributed in an upward parabolic pattern in the bottom layer and its FE result. (b) Drop-shaped pattern generated by swelling a structure that swelling ratio is distributed in a downward parabolic pattern in the bottom layer and its FE result. 
a

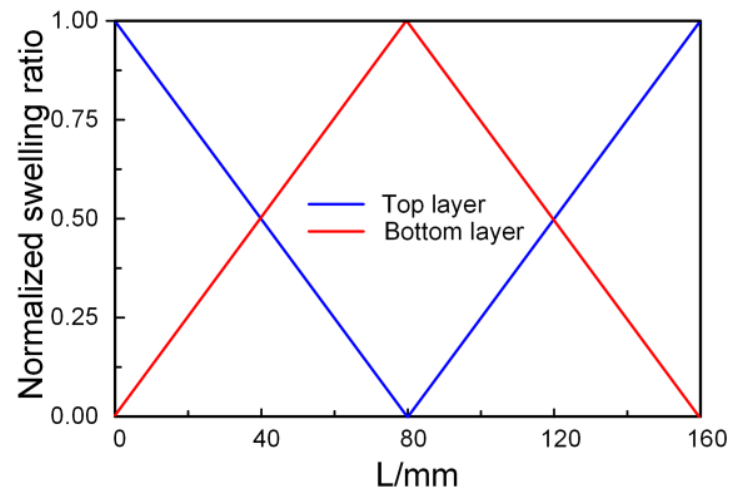

b

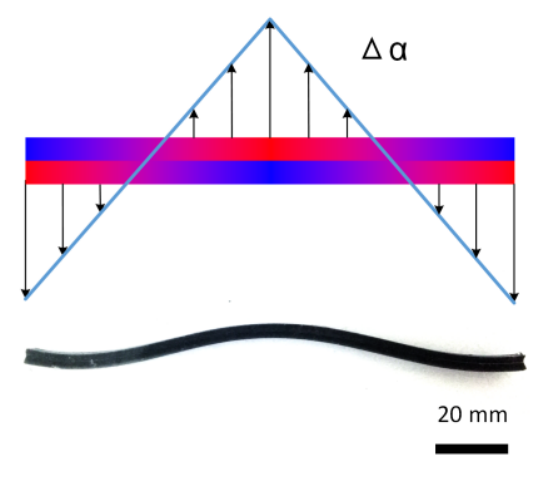

Figure S10. (a) A bilayer structure that the swelling ratios of two layers are distributed in opposite zigzag patterns. (b) The differential swelling strain $(\Delta \alpha)$ go through two changes in direction, resulting in a two-commutated wave-shaped transformation.
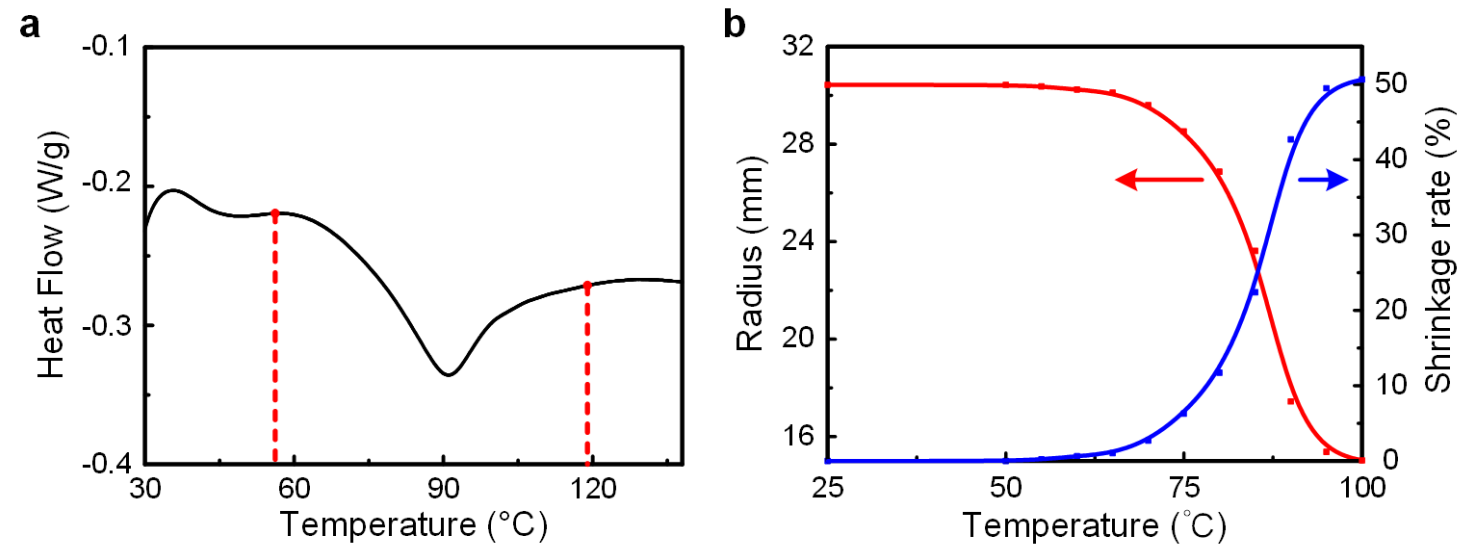

Figure S11. (a) DSC scans of SMP samples showing broad glass transition. (b) The radius and shrinkage of SMP samples measured at different temperatures. 
a

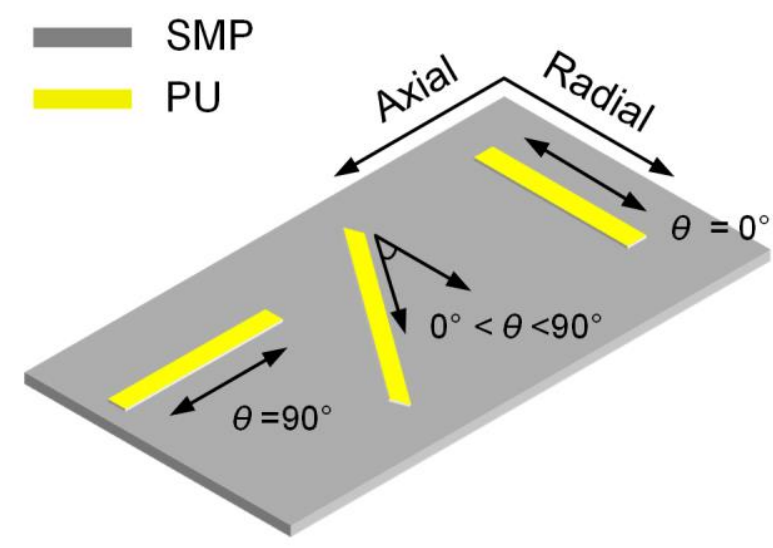

b

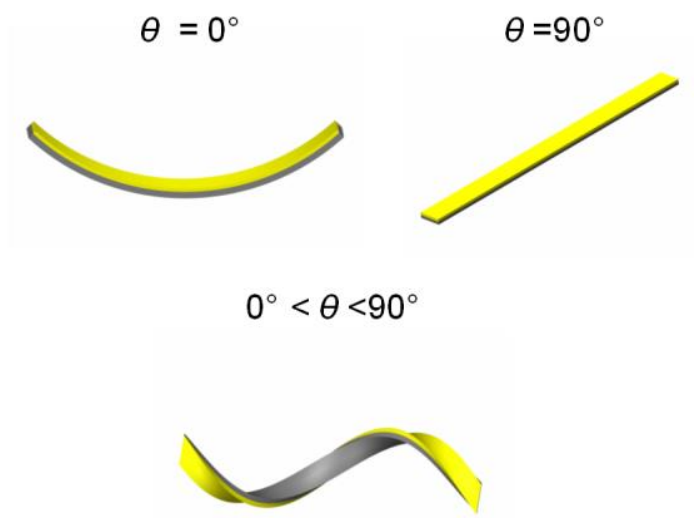

Figure S12. (a) Schematic of PU/SMP bilayer structures preparation with different printing angles. (b) Different deformation modes of PU/SMP bilayer structures caused by the anisotropic shrinkage of SMP layer.

a
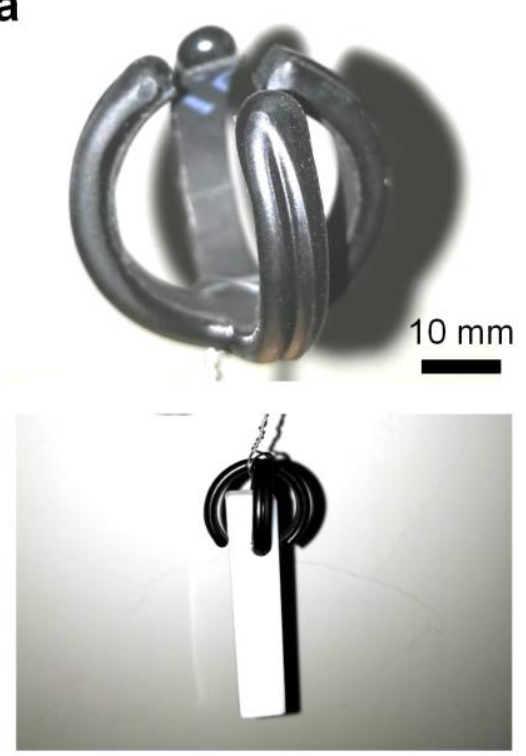

Temperature b
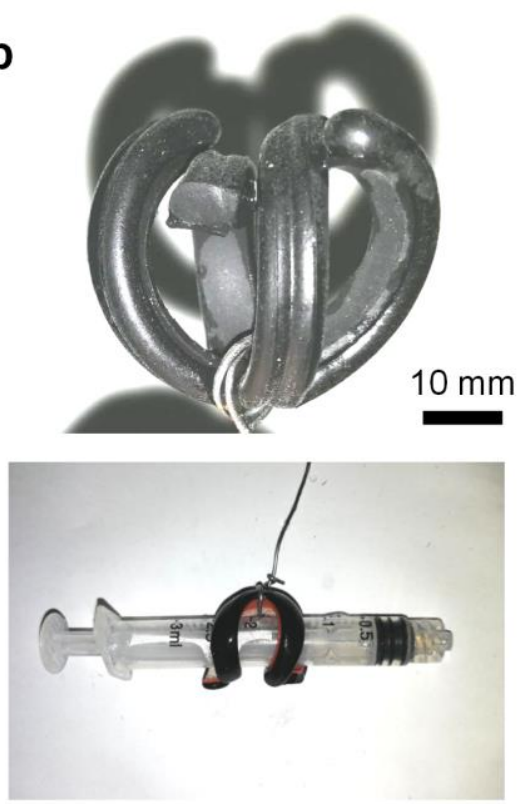

Humidity + Temperature $\left(75^{\circ} \mathrm{C}\right)$

Figure S13. Comparison of deformed shape and gripping mode of temperature-induced deformation (a) and temperature/humidity co-deformation (b). 

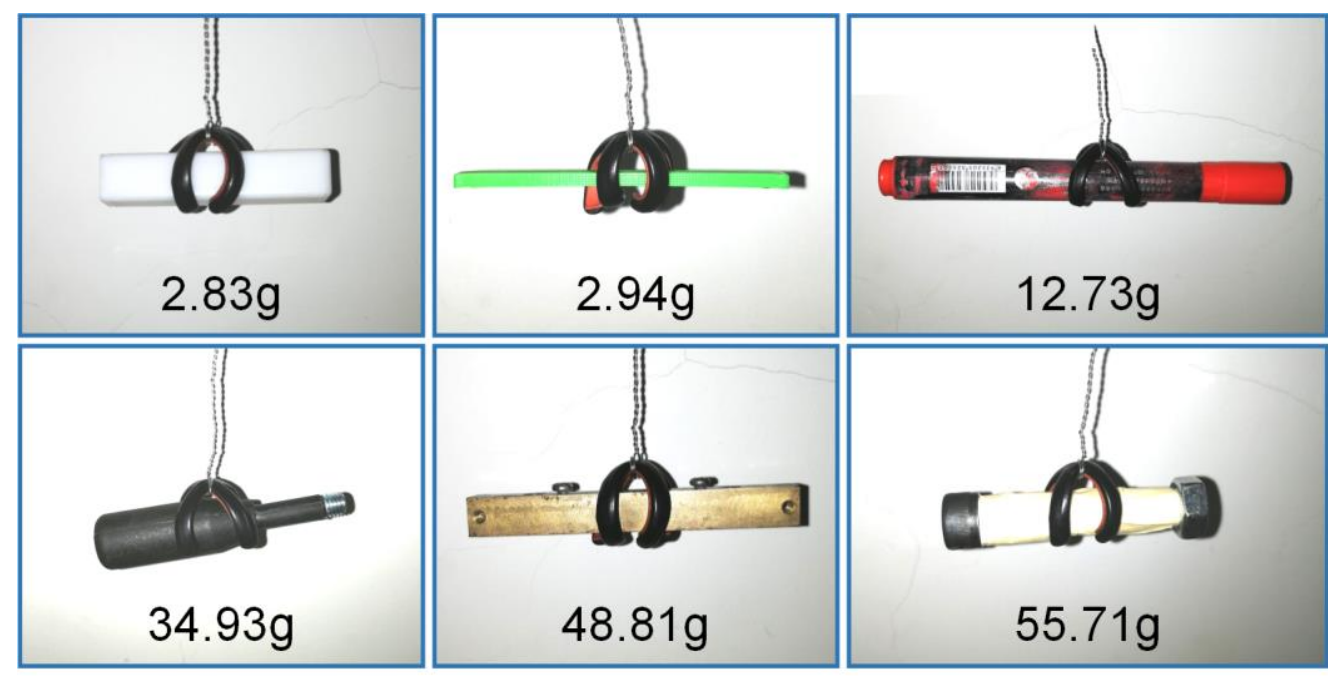

Figure S14. Photographs showing a variety of long objects with different cross-sectional shapes (circles, rectangles, squares, etc.) and different weights can be picked up by the prepared soft gripper.

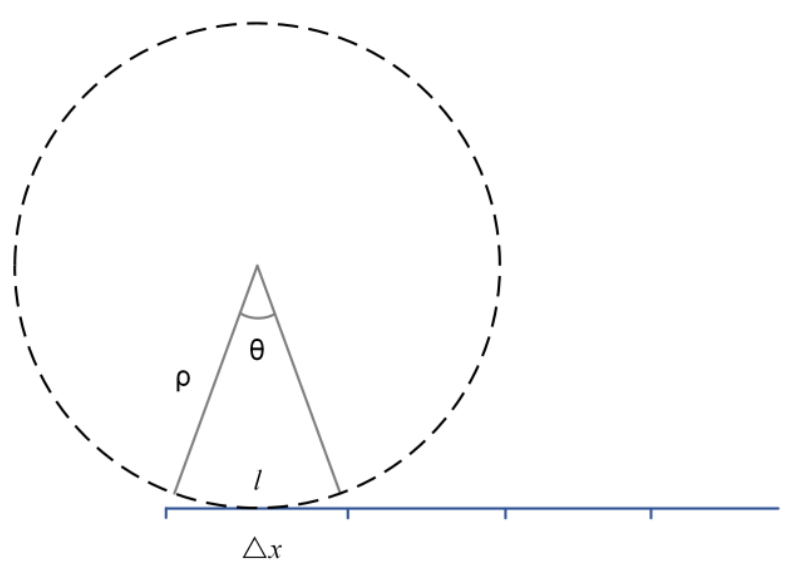

Figure S15. Schematic diagram for calculating radian values of an circle arc formed by an infinitely small interval. In this case, $l$ and $\Delta x$ are considered equal to each other. 


\section{Supporting Movie}

Movie S1

Shape-morphing process of the printed PU-paper bilayer structure having a PU filament with a linear gradient swelling ratio in water

Movie S2

Shape-morphing process of the bilayer PU strip with a linearly decreasing swelling ratio from maximum to $60 \%$ of maximum in the top layer and a linearly decreasing swelling ratio from $50 \%$ of maximum to $10 \%$ of maximum in the bottom layer

Movie S3

Shape-morphing process of the bilayer PU strip with a linearly decreasing swelling ratio in the top layer and linearly increasing swelling ratio in the bottom layer

Movie S4

Shape-morphing process of the bilayer PU strip with a linearly decreasing swelling ratio in the top layer and the right increasing part of upward parabolic style in the bottom layer

Movie S5

Shape-morphing process of the bilayer PU strip with a linearly decreasing swelling ratio in the top layer and a downward parabolic swelling ratio in the bottom layer

Movie S6 
Shape-morphing process of the printed bilayer PU rectangle sheet with a saddle-like swelling ratio distribution in the top layer and a vertically flip-over saddle-like distribution in the bottom layer

Movie S7

Shape-morphing process of the bilayer PU sheet with linearly increasing swelling ratio diagonally distributed from top left to bottom right in the top layer and conversely distributed from bottom right to top left in the bottom layer

Movie S8

Shape-morphing process of the bilayer PU sheet with linearly increasing swelling ratio diagonally distributed from top left to bottom right in the top layer and from bottom left to top right in the bottom layer

Movie S9

Video of grasping a bolt weighing up to $87 \mathrm{~g}$ with the cross-shaped soft gripper. 